cases, however, scenes may share the same set of objects and may only be discriminable on the basis of spatial relations between objects (for example, parking lot versus highway) or on the basis of global scene characteristics (for example, mountain versus desert). MacEvoy and Epstein ${ }^{1}$ propose that an additional pathway, which includes the PPA, may support recognition of scenes based on global scene properties. This dual-pathway hypothesis is consistent with recent studies that found that the PPA contains information about the spatial layout of scenes ${ }^{8,9}$, whereas objectselective cortex contains information about within-scene objects ${ }^{7,9}$. At a behavioral level, coherent scene context facilitates the perception of within-scene objects ${ }^{12,13}$, and objects facilitate scene recognition when these are mutually coherent ${ }^{13}$. A critical question then is where and how the two proposed pathways interact to support scene recognition. In particular, does lateral occipital cortex represent objects independently of scene context or does it integrate object representations with contextual information (Fig. 1)?

Owing to capacity limitations, the brain cannot possibly represent all of the information that is typically present in our cluttered natural environment. In daily life, we overcome such limitations by using cognitive 'top-down' mechanisms. Such goal-driven mechanisms may enhance the processing of relevant information and filter out irrelevant information. For example, we actively search for objects by forming mental templates of the things that we are looking for (such as a friend in a crowd of people), and we make predictions about the presence or absence of objects on the basis of typical scene characteristics (for example, we anticipate a bathtub when we enter a bathroom). Accordingly, previous research has shown that the processing of real-world scenes is biased toward relevant objects $^{3}$ and that this selection is mediated by anticipatory processes ${ }^{4}$. In contrast, MacEvoy and Epstein's results ${ }^{1}$ suggest that the averaging of neural responses evoked by multiple objects in lateral occipital cortex is a highly automatic and 'bottom-up' process, given that participants were not instructed to attend to any particular object in the rapidly presented scenes. Thus, the object-based channel for scene recognition might operate independently of top-down sources that provide feedback to the object recognition system. Alternatively, it is possible that the repeated presentation of a relatively small number of scene types and diagnostic objects may have prompted participants to anticipate these objects while viewing the scenes. An important question for future studies is to address how many within-scene objects can be processed in parallel without prior knowledge of the scenes and objects that will be presented ${ }^{14}$.
In sum, MacEvoy and Epstein ${ }^{1}$ showed that the response in lateral occipital cortex to multiple within-scene objects can be approximated by a linear combination of responses to these objects in isolation. This raises the intriguing possibility that lateral occipital cortex constitutes a pathway involved in scene recognition based on a scene's component objects.

\section{COMPETING FINANCIAL INTERESTS}

The authors declare no competing financial interests.

1. MacEvoy, S.P. \& Epstein, R.A. Nat. Neurosci. 14, 1323-1329 (2011).

2. Thorpe, S., Fize, D. \& Marlot, C. Nature 381, 520-522 (1996).

3. Peelen, M.V., Fei-Fei, L. \& Kastner, S. Nature 460, 94-97 (2009).

4. Peelen, M.V. \& Kastner, S. Proc. Natl. Acad. Sci. USA 108, 12125-12130 (2011).

5. Haxby, J.V. et al. Science 293, 2425-2430 (2001).

6. Epstein, R. \& Kanwisher, N. Nature 392, 598-601 (1998).

7. Goh, J.O. et al. J. Neurosci. 24, 10223-10228 (2004)

8. Kravitz, D.J., Peng, C.S. \& Baker, C.I. J. Neurosci. 31, 7322-7333 (2011).

9. Park, S., Brady, T.F., Greene, M.R. \& Oliva, A. J. Neurosci. 31, 1333-1340 (2011).

10. Walther, D.B., Chai, B., Caddigan, E., Beck, D.M. \& Fei-Fei, L. Proc. Natl. Acad. Sci. USA 108, 9661-9666 (2011).

11. MacEvoy, S.P. \& Epstein, R.A. Curr. Biol. 19, 943-947 (2009).

12. Biederman, I., Mezzanotte, R.J. \& Rabinowitz, J.C. Cognit. Psychol. 14, 143-177 (1982).

13. Davenport, J.L. \& Potter, M.C. Psychol. Sci. 15 , 559-564 (2004).

14. Evans, K.K., Horowitz, T.S. \& Wolfe, J.M. Psychol. Sci. 22 , 739-746 (2011).

\title{
Maintaining a Highwire act
}

In all organisms with a nervous system, the construction of functional neural circuits requires a precise choreography of developmental events that includes the growth and guidance of axons to their proper targets and the formation of mature synapses. Studies in species ranging from worms to mice have revealed that proteins from the PHR (Pam/Highwire/RPM-1) family of E3 ubiquitin ligases modulate neural development via the formation of an F-box protein-containing complex and activation of the MAP kinase signaling cascade. Despite these advances in our understanding of the components and activities of these integral protein complexes, it is still unclear as to how the essential PHR proteins themselves are regulated during development. On page 1267, Tian and colleagues identify Rael as a binding partner of the Drosophila PHR protein Highwire (Hiw) that acts to prevent its degradation and promote refinement of the presynaptic terminal.

The authors identified Rael as a Hiw complex-associating protein using tandem affinity purification and liquid chromatography-tandem mass spectrometry. Flies expressing only a mutant form of Rael exhibited a concurrent increase in neuromuscular junction synaptic bouton number and a decrease in bouton size, a phenotype that mimics that observed in hiw null mutants. Notably, mutation of Rael also resulted in a decrease in the neuronal levels of Hiw protein (but not mRNA), suggesting that Rae1 acts to prevent post-translational degradation of Hiw. Indeed, blocking autophagy was sufficient to rescue both Hiw protein levels and synaptic bouton overgrowth even in the presence of mutated Rae1, whereas

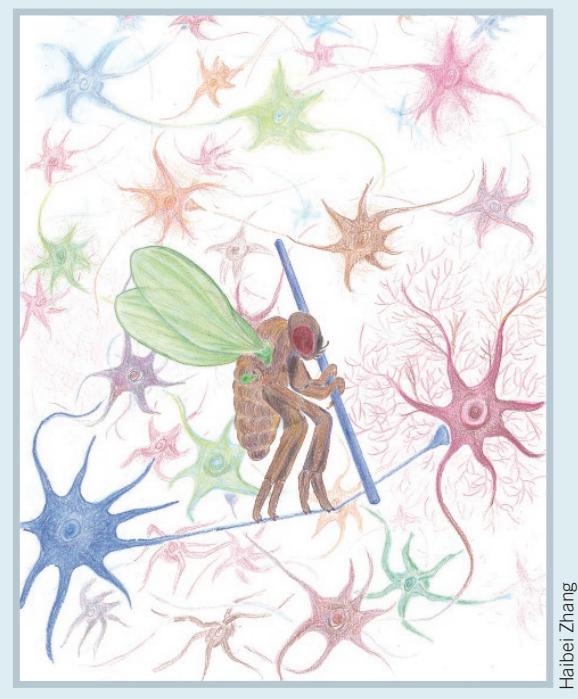
overexpression of wild-type Rael blocked Hiw degradation in the presence of the autophagy-promoting protein Atg1. Taken together, these results suggest that Rae1 is an integral member of the PHR E3 ubiquitin ligase complex that acts to promote synaptic refinement by maintaining Hiw protein levels during development.

Timothy Spencer 
ER R ATA

\section{Erratum: Maintaining a Highwire act}

Timothy Spencer

Nat. Neurosci. 14, 1226 (2011); published online 27 September 2011; corrected after print 27 September 2011

In the version of this article initially published, the artist credit was omitted. The artist's name is Haibei Zhang. The error has been corrected in the HTML and PDF versions of the article. 